\title{
Implications of energy conditions on standard static space-times
}

\author{
Fernando Dobarro ${ }^{\mathrm{a}, *}$, Bülent Ünal ${ }^{\mathrm{b}}$ \\ a Dipartimento di Matematica e Informatica, Università degli Studi di Trieste, Via Valerio 12/B, I-34127 Trieste, Italy \\ ${ }^{\mathrm{b}}$ Department of Mathematics, Bilkent University, Bilkent, 06800 Ankara, Turkey
}

\section{A R T I C L E I N F O}

\section{Article history:}

Received 9 January 2009

Accepted 15 April 2009

\section{MSC: \\ $53 \mathrm{C} 21$ \\ $53 \mathrm{C} 50$ \\ $53 \mathrm{C} 80$}

\section{Keywords:}

Warped products

Standard static space-times

Energy conditions

Sub-harmonic functions

Scalar curvature map

Conformal hyperbolicity

Conjugate points

\begin{abstract}
A B S T R A C T
In the framework of standard static space-times, we state a family of sufficient or necessary conditions for a set of physically reasonable energy and convergence conditions in relativity and related theories. We concentrate our study on questions about the sub-harmonicity of the warping function, the scalar curvature map, conformal hyperbolicity, conjugate points and the time-like diameter of this class of space-times.
\end{abstract}

(C) 2009 Elsevier Ltd. All rights reserved.

\section{Introduction}

This paper deals with the study of energy conditions on standard static space-times. Our first objective is to obtain a family of necessary and/or sufficient conditions for a set of energy conditions on standard static space-times. The second aim is to apply the latter to a group of questions about conformal hyperbolicity (in the sense of M.J. Markowitz) and the existence of conjugate points in the same framework. Especially, we also devote part of our study to consider a set of partial differential operators involved in these discussions.

Warped products (in particular Lorentzian ones) have played a central role in general relativity in the construction of general solutions of the Einstein field equation for a long period of time [1,2].

A standard static space-time (also said to be globally static, see [3]) is a Lorentzian warped product where the warping function is defined on a Riemannian manifold (called the natural space or Riemannian part) and acting on the negative definite metric on an open interval of real numbers (see Definition 2.2). This structure can be considered as a generalization of the Einstein static universe. In [2], it was shown that any static space-time ${ }^{1}$ is locally isometric to a standard static.

In general relativity and related theories, the energy conditions are a set of physically reasonable imposed constraints on the underlying space-time (in-depth discussions about these conditions can be found in [7-9,2,10,11] among many others). In $[12,13]$, the author investigates conditions on the warping function which guarantee that the underlying standard static

\footnotetext{
* Corresponding author. Fax: +39 0405582636.

E-mail addresses: dobarro@dmi.units.it (F. Dobarro), bulentunal@mail.com (B. Ünal).

1 An $n$-dimensional space-time $(M, g)$ is said to be static if there exists a nowhere vanishing time-like Killing vector field $X$ on $M$ such that the distribution of each $(n-1)$-plane orthogonal to $X$ is integrable (see [1, Subsection 3.7] and also the general relativity texts [4-6]).
} 
space-times either satisfy or else fail to satisfy certain energy or convergence conditions. Some of our results in the following sections are narrowly related to those of D. Allison.

We now provide an outline of the paper.

In Section 2, we recall some definitions and suitable expressions of Ricci and scalar curvatures for a standard static space-time.

Sections 3 and 4 contain the principal new results of this article. Some of them were already announced (without proofs) in the survey work [14].

In Subsection 3.1 we introduce the energy and convergence conditions and make some generic comments about them and their physical applications. In the first part of Subsection 3.2, we state a family of results that show up a connection between the strong energy condition and a family of Liouville type results for sub-harmonic functions on the Riemannian part of a standard static space-time (see Theorem 3.1 and its corollaries). In the second part, the principal results are Theorems 3.7, 3.11, 3.12, 3.15 and their corollaries; notice particularly Corollary 3.9. All these results are based on suitable hypotheses for the definiteness of the quadratic forms associated to a family of two covariant tensors involving the metric, Hessian and Ricci tensors on the natural space. Unlike Theorems 3.7 and 3.11, Theorems 3.12 and 3.15 are new results, i.e., they were announced nowhere, but their proofs follow the same order of ideas. At the end of this subsection, in Theorem 3.19, we give a "partial" extension of a Bourguignon/Fischer-Marsden result stated in [15, p. 228-230]/[16] to the case where the involved manifold is complete but noncompact and with nonnegative Ricci curvature. The statements of the latter and the results connected with the Liouville type properties were not included in [14].

In the main Section 4, we combine the results of Section 3 (particularly, Theorem 3.7) with those of Markowitz in [17].

In Subsection 4.1, we obtain sufficient conditions for a standard static space-time to have trivial Lorentzian pseudodistance or to be conformally hyperbolic in the sense of Markowitz (see Theorems 4.1 and 4.2 respectively).

In Subsection 4.2, we establish sufficient conditions in order to guarantee that any causal geodesic on a standard static space-time has a pair of conjugate points. By a stronger set of hypotheses, we also obtain an estimate from above for the time-like diameter of the standard static space-time (see Corollaries 4.3 and 4.4).

Finally, in Subsection 4.3, we show some results connecting the tensor $Q_{g_{F}}^{f}$ (see (2.3)), conformal hyperbolicity, concircular scalar fields and Hessian manifolds. More precisely, we give sufficient conditions for a standard static space-time to be conformally hyperbolic where the Riemannian part admits a concircular scalar field or is a globally Hessian manifold.

\section{Preliminaries}

Throughout the article $I$ will denote an open real interval of the form $I=\left(t_{1}, t_{2}\right)$ where $-\infty \leq t_{1}<t_{2} \leq \infty$. Moreover, $\left(F, g_{F}\right)$ will denote a connected Riemannian manifold without boundary with $\operatorname{dim} F=s$. Finally, on an arbitrary differentiable manifold $N, C_{>0}^{\infty}(N)$ denotes the set of all strictly positive $C^{\infty}$ functions defined on $N, T N=\bigcup_{p \in N} T_{p} N$ denotes the tangent bundle of $N$ and $\mathfrak{X}(N)$ will denote the $C^{\infty}(N)$-module of smooth vector fields on $N .^{2}$

For completeness we recall the general definition of a singly warped product.

Definition 2.1. Let $\left(B, g_{B}\right)$ and $\left(N, g_{N}\right)$ be pseudo-Riemannian manifolds and $b \in C_{>0}^{\infty}(B)$. Then the (singly) warped product $B \times{ }_{b} N$ is the product manifold $B \times N$ furnished with the metric tensor

$$
g=\pi^{*}\left(g_{B}\right) \oplus(b \circ \pi)^{2} \sigma^{*}\left(g_{N}\right),
$$

where $\pi: B \times N \rightarrow B$ and $\sigma: B \times N \rightarrow N$ are the usual projection maps and ${ }^{*}$ denotes the pull-back operator on tensors.

Definition 2.2. Let $f \in C_{>0}^{\infty}(F)$. The $n(=1+s)$-dimensional product manifold $I \times F$ furnished with the metric tensor $g=-f^{2} \mathrm{~d} t^{2} \oplus g_{F}$ is called a standard static space-time (also usually said to be globally static; see [3]) and is denoted by $I_{f} \times F$.

On a warped product of the form $B \times_{f} F$, we will denote the set of lifts to the product by the corresponding projection of the vector fields in $\mathfrak{X}(B)$ (respectively, $\mathfrak{X}(F)$ ) by $\mathfrak{L}(B)$ (respectively, $\mathfrak{L}(F)$ ) (see [2]). We will use the same symbol for a tensor field and its lift.

The following formula of the curvature Ricci tensor can be easily obtained from $[1,18,19,2]$.

Proposition 2.3. Let $I_{f} \times F$ be a standard static space-time furnished with the metric $g=-f^{2} \mathrm{~d} t^{2} \oplus g_{F}$. Suppose that $U_{1}, U_{2} \in \mathfrak{L}(I)$ and $V_{1}, V_{2} \in \mathfrak{L}(F)$. If Ric and $\operatorname{Ric}_{g_{F}}$ denote the Ricci tensors of $I_{f} \times F$ and $\left(F, g_{F}\right)$, respectively, then

$$
\begin{aligned}
\operatorname{Ric}\left(U_{1}+V_{1}, U_{2}+V_{2}\right) & =\operatorname{Ric}_{g_{F}}\left(V_{1}, V_{2}\right)+f \Delta_{g_{F}} f \mathrm{~d} t^{2}\left(U_{1}, U_{2}\right)-\frac{1}{f} \mathrm{H}_{g_{F}}^{f}\left(V_{1}, V_{2}\right) \\
& =-\frac{1}{f} \mathscr{L}_{g_{F}}^{*} f\left(V_{1}, V_{2}\right)-\frac{1}{f} \Delta_{g_{F}} f g\left(U_{1}+V_{1}, U_{2}+V_{2}\right)
\end{aligned}
$$

\footnotetext{
2 Notation: In the present study, manifolds are denoted by $B, F, M, N$; points by $p, q, x, y$; vectors by v, w, x, y and also vector fields by $V, W, X, Y$.
} 
where $\mathrm{H}_{g_{F}}^{f}$ is the Hessian tensor of $f$ on $\left(F, g_{F}\right), \Delta_{g_{F}}(\cdot)=g_{F}^{i j} \nabla_{i}^{g F} \nabla_{j}^{g F}(\cdot)$ is the Laplace-Beltrami operator and $\mathscr{L}_{g_{F}}^{*} f$ is the 2covariant tensor given by

$$
\mathscr{L}_{g_{F}}^{*} f:=-f \text { Ric }_{g_{F}}-\Delta_{g_{F}} f g_{F}+\mathrm{H}_{g_{F}}^{f}
$$

on $\left(F, g_{F}\right)$.

In [18], we studied the problem of constancy of the scalar curvature on a standard static space-time; in particular we obtained the following formula.

Proposition 2.4. Let $I_{f} \times F$ be a standard static space-time furnished with the metric $g=-f^{2} \mathrm{~d} t^{2} \oplus g_{F}$. If $\tau$ and $\tau_{g_{F}}$ denote the scalar curvatures of $I_{f} \times F$ and $\left(F, g_{F}\right)$, respectively, then

$$
\tau=\tau_{g_{F}}-2 \frac{1}{f} \Delta_{g_{F}} f
$$

As a consequence, $f$ is sub-harmonic (i.e., $\Delta_{g_{F}} f \geq 0$ ) if and only if $\tau<\tau_{g_{F}}$ (since $f \in C_{>0}^{\infty}(F)$ ).

Now, we will introduce a special tensor frequently used to establish energy conditions on a standard static space-time (see for instance $[12,13]$ ). This tensor will be play a central role in the following sections.

Notation 2.5. Let $\left(F, g_{F}\right)$ be a Riemannian manifold and $f \in C_{>0}^{\infty}(F)$. From now on, $Q_{g_{F}}^{f}$ denotes the 2-covariant tensor given by

$$
Q_{g_{F}}^{f}:=\Delta_{g_{F}} f g_{F}-H_{g_{F}}^{f} .
$$

If it is necessary, we will emphasize the evaluation points $p \in F$ by writing $Q_{g_{F} \mid p}^{f}$. We will apply the latter convention for other tensors too.

Furthermore, we will denote its associated quadratic form by $\mathcal{Q}_{g_{F}}^{f}$ for any point $p \in F$. More precisely, for any $\mathrm{v} \in \mathrm{T}_{p} F$

$$
Q_{g_{F} \mid p}^{f}(\mathrm{v}):=Q_{g_{F} \mid p}^{f}(\mathrm{v}, \mathrm{v}),
$$

where $\mathrm{T}_{p} F$ is the tangent space to $F$ at $p$.

Analogously, we will denote the associated quadratic form to the Ricci tensor Ric by Ric. So, for any unit tangent vector $\mathrm{v} \in \mathrm{T}_{p} F$

$$
\operatorname{Ric}_{g_{F} \mid p}(\mathrm{v}):=\operatorname{Ric}_{g_{F} \mid p}(\mathrm{v}, \mathrm{v})
$$

is the so-called Ricci curvature in the direction of $v$ (see [20-22]).

Besides, we will denote the associated quadratic form to the 2 -covariant tensor $\mathscr{L}_{g_{F}}^{*} f$ by $\mathcal{L}_{g_{F}}^{*} f$.

Remark 2.6. ${ }^{3}$ It is easy to prove that the 2-covariant tensor $Q_{g_{F}}^{f}$ is divergence-free. Indeed, it is sufficient to apply the definition of the involved differential operators, the identity $\operatorname{div}_{g_{F}}\left(\phi g_{F}\right)=\mathrm{d} \phi$ for all $\phi \in C^{\infty}(F)$ and commute derivatives (see [2, p. 85-87]).

Remark 2.7. Let $Q_{g_{F}}^{f}$ be defined as above with $\operatorname{dim} F=s \geq 2$.

(i) If the symmetric bilinear form $Q_{g_{F} \mid p}^{f}$ is positive (respectively, negative) semi-definite for a point $p \in F$, then ( $\left.\Delta_{g_{F}} f\right)_{\mid p} \geq 0$ (respectively, $\leq 0)$. It is sufficient to observe that $\operatorname{tr}_{g_{F}} Q_{g_{F}}^{f}=(s-1) \Delta_{g_{F}} f$ and the $g_{F}$-trace preserves the sign.

(ii) It is clear that (i) holds while replacing semi-definiteness by definiteness and accordingly, $\geq$ by $>$.

(iii) Assume now that $F$ is compact and $f: F \rightarrow \mathbb{R}$ is smooth. The so-called Bochner lemma (see [23, p. 39]) says: if $\Delta_{g_{F}} f \geq 0$, then $f$ is constant. Thus, it just follows from (ii) that $f$ is constant if $Q_{g_{F}}^{f}$ is positive semi-definite.

Furthermore, if $Q_{g_{F}}^{f}$ is negative semi-definite, then $Q_{g_{F}}^{-f}=-Q_{g_{F}}^{f}$ is positive semi-definite; so $-f$ (and obviously $f$ ) is constant.

Remark 2.8. Notice that applying (2.3), the $(0,2)$-tensor $\mathscr{L}_{g_{F}}^{*} f$ defined in $(2.2)$ takes the form

$$
\mathscr{L}_{g_{F}}^{*} f:=-f \text { Ric }_{g_{F}}-Q_{g_{F}}^{f} .
$$

This tensor is strongly associated to the scalar curvature map between Banach manifolds that apply to each metric on a smooth manifold (eventually, to a sub-domain) the corresponding scalar curvature. Indeed, if $g_{F}$ is a suitable Riemannian

\footnotetext{
3 We observe that this result is valid on any pseudo-Riemannian manifold if one takes the signature of the metric in the definition of the divergence into account.
} 
metric on such a manifold and $f$ is a sufficiently regular function on the manifold, $\mathscr{L}_{g_{F}}^{*} f$ is the $L^{2}$-formal adjoint of the linearized scalar curvature operator. There is a large literature about these operators; for instance [24-26,15,27,16,28,3, 29-37] and the many references therein.

The kernel of the operator (2.6) will play an important role in our discussion about the dominant energy condition in the following section.

\section{Energy conditions and implications}

There are several natural energy conditions considered in general relativity and cosmology questions (see [1,7-9,2,10,11] among others). They are related for instance with: space-time singularities (see [38]), existence of conjugate points (see [39]), splitting theorems and existence of time-like lines (see [40]), Lorentzian wormholes (see [10]), higher-dimensional black holes (see [41-43]), space-like foliations (see [44]), dS/CFT and space-time topology (see [45]) among many other topics.

In the first subsection we will introduce some energy conditions on generic space-times, while in the second subsection we will state some of these for a standard static space-time of the form $M=I_{f} \times F$ with the metric $g=-f^{2} \mathrm{~d} t^{2} \oplus g_{F}$ in terms of the tensors $\mathscr{L}_{g_{F}}^{*} f$ and $Q_{g_{F}}^{f}$ and analyze possible consequences of these conditions.

\subsection{Definitions, generalities and more}

In this subsection, let us consider a space-time $\left(N, g_{N}\right)$ of dimension $n \geq 3$.

Assuming the conventions in $[13,46,47]$; the space-time $\left(N, g_{N}\right)$ is said to satisfy the strong energy condition (respectively, reverse strong energy condition), SEC for short (respectively, $\mathrm{RSEC})$, if $\operatorname{Ric}(\mathrm{x}, \mathrm{x}) \geq 0$ (respectively, Ric $(\mathrm{x}, \mathrm{x}) \leq 0)$ for all causal tangent vectors $\mathrm{X}$.

Furthermore $\left(N, g_{N}\right)$ is said to satisfy the time-like (respectively null, space-like) convergence condition, TCC for short (respectively NCC, SCC), if Ric $(\mathrm{x}, \mathrm{x}) \geq 0$ for all time-like (respectively null, space-like) tangent vectors $\mathrm{x}$. The corresponding reverses (i.e., $\operatorname{Ric}(\mathrm{x}, \mathrm{x}) \leq 0$ ) will be denoted as above by $\mathrm{RTCC}, \mathrm{RNCC}$ and $\mathrm{RSCC}$, respectively.

Notice that the SEC implies the NCC. Furthermore the TCC is equivalent to the SEC, by continuity. The actual difference between TCC and SEC follows from the fact that while TCC is just a geometric condition imposed on the Ricci tensor, SEC is a condition on the stress-energy tensor. They can be considered equivalent due to the Einstein equation (see (3.1) and the discussion about the SEC definition adopted by us and that in the sense of Hawking and Ellis). ${ }^{4}$

Moreover, a space-time is said to satisfy the weak energy condition, WEC for short, if $\mathrm{T}(\mathrm{x}, \mathrm{x}) \geq 0$ for all time-like vectors, where $\mathrm{T}$ is the energy-momentum tensor, which is determined by physical considerations.

In this article, when we consider the energy-momentum tensor, we assume that the Einstein equation holds (see [9,2]). More explicitly,

$$
\text { Ric }-\frac{1}{2} \tau g_{N}=8 \pi \mathrm{T}
$$

Notice that in particular, (3.1) gives the explicit form of the energy-momentum tensor T.

The WEC has many applications in general relativity theory such as nonexistence of closed time-like curves (see [48]) and the problem of causality violation [49]. But its fundamental use still lies in Penrose's singularity theorem (see [50]).

Notice that the notion of SEC considered in our study is not the same one stated in [9, p. 95] (see also [1, p. 434], [51]). Indeed for Hawking and Ellis, $\left(N, g_{N}\right)$ verifies the strong energy condition (HE-SEC for short) if and only if

$$
\mathrm{T}(\mathrm{x}, \mathrm{x}) \geq \frac{1}{2} \operatorname{tr} \mathrm{T}_{N}(\mathrm{x}, \mathrm{x}),
$$

for all time-like tangent vectors $\mathrm{x}$.

On the other hand, by (3.1)

$$
\frac{2-n}{2} \tau=8 \pi \operatorname{tr} \mathrm{T} \text {. }
$$

Thus

$$
\text { Ric }=8 \pi\left[\mathrm{T}-\frac{1}{n-2} \operatorname{tr} \mathrm{Tg}_{N}\right] .
$$

So the HE-SEC is equivalent to the SEC, if and only if $n=4$. Furthermore,

\footnotetext{
4 One can refer to [1, p. 434] for further interesting comments about the different definitions and conventions about these energy and convergence conditions.
} 
- if $n>4$ and $\tau \geq 0$, HE-SEC implies SEC;

- if $n>4$ and $\tau \leq 0$, SEC implies HE-SEC;

- if $n \geq 3$ and $\tau=0$, then SEC, HE-SEC and WEC are equivalent.

At this point we immediately observe that neither SEC nor HE-SEC implies WEC (see [10, p. 117] for dimension 4). However,

- SEC + " $\tau \geq 0$ " implies WEC;

- WEC + " $\tau \leq 0$ " implies SEC.

Consequently,

- if $\tau=0$, SEC is equivalent to WEC.

Another important energy condition is the so-called dominant energy condition (DEC for short), namely: WEC + " $\mathrm{T}^{i j} \mathrm{X}_{j}$ is causal for all time-like vectors X". It is clear that DEC implies WEC. This condition is extremely relevant, for instance in the recent studies of higher-dimensional black holes [41-43] (see also [7]).

An equivalent formulation of the DEC is (see $[43,52,45])$ : " $\mathrm{T}(\mathrm{x}, \mathrm{y}) \geq 0$ for all future pointing causal vectors $\mathrm{x}$ and $\mathrm{y}$ ".

\subsection{Some implications on standard static space-times}

Let $M=I_{f} \times F$ be a standard static space-time with the metric $g=-f^{2} \mathrm{~d} t^{2} \oplus g_{F}$. Thus,

$$
g\left(\partial_{t}, \partial_{t}\right)=-f^{2}<0
$$

i.e., $\partial_{t}$ is time-like and by Proposition 2.3,

$$
\operatorname{Ric}\left(\partial_{t}, \partial_{t}\right)=f \Delta_{g_{F}} f .
$$

So we can easily state the following.

Theorem 3.1 ([13]). Let $M=I_{f} \times F$ be a standard static space-time with the metric $g=-f^{2} \mathrm{~d} t^{2} \oplus g_{F}$. If the space-time $(M, g)$ satisfies the SEC (or equivalently, the TCC), then $f$ is sub-harmonic, i.e., $\Delta_{g_{F}} f \geq 0$.

In particular, we have the following:

Corollary 3.2. Let $\left(F, g_{F}\right)$ be admitting no nonconstant sub-harmonic positive function. Then, the SEC is verified by no standard static space-time with "natural space $\left(F, g_{F}\right)$ and nonconstant warping function".

Corollary 3.3. Let $M=I_{f} \times F$ be a standard static space-time with the metric $g=-f^{2} \mathrm{~d} t^{2} \oplus g_{F}$ satisfying the SEC (or equivalently, the TCC). If $\left(F, g_{F}\right)$ is compact, then $f$ is a positive constant.

Proof. It is sufficient to apply Theorem 3.1 and the Bochner lemma (see Remark 2.7).

Several sufficient conditions are known for nonexistence of a nonconstant nonnegative sub-harmonic function on a complete Riemannian manifold. Thus, again by Theorem 3.1, it is possible to obtain a family of results in the cases where the natural space is a complete but noncompact Riemannian manifold. Here, we will mention some of them based on the results of P. Li, R. Schoen and Shing Tung Yau (see $[53,54]$ and references therein). ${ }^{5}$

Corollary 3.4. Let $M=I_{f} \times F$ be a standard static space-time with the metric $g=-f^{2} \mathrm{~d} t^{2} \oplus g_{F}$ satisfying the SEC (or equivalently, the TCC), where $\left(F, g_{F}\right)$ is a noncompact complete Riemannian manifold. Suppose that $x_{0} \in F$ is a fixed point and $\rho$ denotes the distance function to $x_{0}$ in $\left(F, g_{F}\right)$. Then the warping function $f$ is constant and $\left(F, g_{F}\right)$ has finite volume if at least one of the following conditions is verified:

(1) $f \in L^{p}(F)$ for some $p>1$.

(2) $f \in L^{1}(F)$ and there exist constants $C>0$ and $\alpha>0$ such that the Ricci curvature of $\left(F, g_{F}\right)$ satisfies Ric $_{g_{F} \mid x} \geq-C(1+$ $\left.\rho^{2}(x)\right)\left(\log \left(1+\rho^{2}(x)\right)\right)^{-\alpha}$.

(3) $f \in L^{p}(F)$ for some $0<p<1$ and there exists a constant $\delta(s)>0$ depending only on s, such that the Ricci curvature of $\left(F, g_{F}\right)$ satisfies $\operatorname{Ric}_{g_{F} \mid x} \geq-\delta(s) \rho^{-2}(x)$ as $x \rightarrow \infty$.

(4) $f \in L^{1}(F)$ and there exists a constant $C>0$ such that the Ricci curvature of $\left(F, g_{F}\right)$ satisfies Ric $_{g_{F} \mid x} \geq-C\left(1+\rho^{2}(x)\right)$.

Proof. Notice that $f$ is positive and Theorem 3.1 says that $f$ is sub-harmonic. Thus, we have the following reasonings.

(1) It is sufficient to apply [53, Theorem 12.1] (see also [55]).

(2) It is sufficient to apply [53, Theorem 12.2] (see also [56]).

\footnotetext{
${ }^{5}$ From now on $L^{p}(F)$ denotes the usual norm space of $p$-integrable functions on a manifold $F$, where $p$ is a real number.
} 
(3) It is sufficient to apply [53, Theorem 12.3] (see also [56]).

(4) It is sufficient to apply [53, Theorem 12.4] (see also [57]).

In order to state the next corollary, we recall that a complete Riemannian manifold is called a Cartan-Hadamard manifold if it is simply connected and has nonpositive sectional curvature (see [54, p. 128]).

Corollary 3.5. Let $M=I_{f} \times F$ be a standard static space-time with the metric $g=-f^{2} \mathrm{~d} t^{2} \oplus g_{F}$ satisfying the SEC (or equivalently, the TCC), where $\left(F, g_{F}\right)$ is a complete noncompact Riemannian manifold. Assume that $\left(F, g_{F}\right)$ satisfies one of the following conditions:

(1) $\left(F, g_{F}\right)$ is a Cartan-Hadamard manifold.

(2) $\left(F, g_{F}\right)$ has Ricci curvature bounded from below and the volume of every unit geodesic ball is uniformly bounded from below.

Then, the warping function $f \in L^{p}(F)$ for some $0<p \leq 1$ only if $f$ is constant.

Proof. As above, $f$ is positive, so Theorem 3.1 says that $f$ is sub-harmonic. Hence, it is sufficient to apply [53, Theorem 12.5] (see also [56]).

Corollary 3.6. Let $M=I_{f} \times F$ be a standard static space-time with the metric $g=-f^{2} \mathrm{~d} t^{2} \oplus g_{F}$ satisfying the SEC (or equivalently, the TCC), where $\left(F, g_{F}\right)$ is a complete noncompact Riemannian manifold. Thus, if the $L^{p}$-norm of the warping function $f$ satisfies

$$
\int_{B_{x}(r)} f^{p}=o\left(r^{2}\right)
$$

as $r \rightarrow \infty$ for some fixed point $x \in F$, then the warping function $f$ is constant and

$$
\limsup _{r \rightarrow \infty} r^{-2} V_{x}(r)=0,
$$

where $B_{x}(r)$ is a geodesic ball in $\left(F, g_{F}\right)$ centered at $x$ of radius $r$ and its volume is given by $V_{x}(r)$.

Proof. Similarly to the proof of Corollary 3.5, the positivity of $f$ and Theorem 3.1 imply that $f$ is sub-harmonic. Thus, it is sufficient to apply [53, Proposition 12.6] (see also [55]).

Now, we state necessary conditions for a standard static space-time to satisfy the NCC and other conditions of the curvature Ricci tensor as in the assumptions of Theorems 5.1 and 5.8 in [17] (for a detailed discussion about the energy conditions see for instance [9, Section 4.3]). These are analog to the more accurate ones in [58, Proposition 4.2] for the generalized Robertson-Walker space-time.

The following result, already partially announced in the survey [14], is a simple consequence of (2.1), Notation 2.5 and Remark 2.7 (see also [13, Theorems 3.3 and 3.6]):

Theorem 3.7 ([14, Thm. 4.1]). Let $M=I_{f} \times F$ be a standard static space-time with the metric $g=-f^{2} \mathrm{~d} t^{2} \oplus g_{F}$, where $s=\operatorname{dim} F \geq 2$.

(1) $\mathcal{L}_{g_{F}}^{*} f$ is negative (respectively, positive) semi-definite if and only if M satisfies the NCC (respectively, RNCC).

(2) If $\mathcal{R i c}_{g_{F}}$ and $\mathcal{Q}_{g_{F}}^{f}$ are positive semi-definite, then M satisfies the TCC and the NCC.

(3) If $\mathcal{R i c}_{g_{F}}$ and $\mathcal{Q}_{g_{F}}^{f}$ are negative semi-definite, then $\operatorname{Ric}(\mathrm{w}, \mathrm{w}) \leq 0$ for any causal vector $\mathrm{w} \in T M$, i.e., M satisfies the RNCC and the RTCC.

(4) If $\left(F, g_{F}\right)$ is Ricci flat, then $Q_{g_{F}}^{f}$ is positive semi-definite if and only if M satisfies the NCC.

Remark 3.8. In order to obtain (1) in Theorem 3.7, it is useful to note that for any $\mathrm{v} \in T F$, the vector field $r f^{-1} \sqrt{g_{F}(\mathrm{v}, \mathrm{v})} \partial_{t}+$ $\mathrm{v} \in T M$ is causal iff $|r| \geq 1$. Moreover the vector field on $M$ is null iff $|r|=1$.

Now, we will deal with the energy conditions in terms of the energy-momentum tensor (see [10] for other results in this direction). Let $M=I_{f} \times F$ be a standard static space-time with the metric $g=-f^{2} \mathrm{~d} t^{2} \oplus g_{F}$. Recall that we assume the validity of the Einstein equation (3.1). On the other hand, tangent vectors $\mathrm{w} \in T\left(I_{f} \times F\right)$ can be decomposed into $\mathrm{w}=\mathrm{u}+\mathrm{v}$ with $\mathrm{u} \in T I$ and $\mathrm{v} \in T F$. It is easy to obtain from (2.1) and Proposition 2.4 that for any $U_{1}, U_{2} \in \mathfrak{X}(I)$ and $V_{1}, V_{2} \in \mathfrak{X}(F)$

$$
\begin{aligned}
8 \pi \mathrm{T}\left(U_{1}+V_{1}, U_{2}+V_{2}\right) & =-\frac{1}{f} \mathscr{L}_{g_{F}}^{*} f\left(V_{1}, V_{2}\right)-\frac{1}{2} \tau_{g_{F}} g\left(U_{1}+V_{1}, U_{2}+V_{2}\right) \\
& =\operatorname{Ric}_{g_{F}}\left(V_{1}, V_{2}\right)+\frac{1}{f} Q_{g_{F}}^{f}\left(V_{1}, V_{2}\right)-\frac{1}{2} \tau_{g_{F}} g\left(U_{1}+V_{1}, U_{2}+V_{2}\right) .
\end{aligned}
$$


In particular, for any $U \in \mathfrak{X}(I)$ and $V \in \mathfrak{X}(F)$, we have

$$
\begin{aligned}
8 \pi \mathrm{T}(U+V, U+V) & =-\frac{1}{f} \mathcal{L}_{g_{F}}^{*} f(V, V)-\frac{1}{2} \tau_{g_{F}} g(U+V, U+V) \\
& =\mathcal{R} i c_{g_{F}}(V)+\frac{1}{f} Q_{g_{F}}^{f}(V)-\frac{1}{2} \tau_{g_{F}} g(U+V, U+V) .
\end{aligned}
$$

So, by (3.4) it results:

Corollary 3.9. Let $M=I_{f} \times F$ be a standard static space-time with the metric $g=-f^{2} \mathrm{~d} t^{2} \oplus g_{F}$. If the space-time (M, $g$ ) satisfies the WEC, then $\tau_{g_{F}} \geq 0$.

Corollary 3.10. Suppose that $\left(F, g_{F}\right)$ is a Riemannian manifold admitting at least one point at which the scalar curvature is negative. Then, the WEC condition cannot be verified by any standard static space-time with natural space $\left(F, g_{F}\right)$.

Recalling that on a Riemannian manifold $\left(F, g_{F}\right)$ of dimension $s$ the scalar curvature $\tau_{g_{F}}(p):=\sum_{j=1}^{s} \operatorname{Ric}_{g_{F} p}\left(e_{j}, e_{j}\right)$, where $\left\{e_{j}\right\}_{j=1}^{s}$ is an arbitrary orthonormal basis for the tangent space $T_{p} F$ (see for instance [21]) and applying (3.9), we obtain the following couple of results when $\operatorname{dim} F \geq 2$.

Theorem 3.11 ([14, Thm. 4.2]). Let $M=I_{f} \times F$ be a standard static space-time with the metric $g=-f^{2} \mathrm{~d} t^{2} \oplus g_{F}$.

(1) If $\mathcal{R i c}_{g_{F}}$ and $\mathcal{Q}_{g_{F}}^{f}$ are positive (respectively, negative) semi-definite, then $\mathrm{T}(\mathrm{w}, \mathrm{w}) \geq 0$ (respectively, $\leq 0$ ) for any causal vector $\mathrm{W} \in T M$.

(2) If $\left(F, g_{F}\right)$ is Ricci flat, then for any $\mathrm{u} \in T I$ and $\mathrm{v} \in T F$, we have $8 \pi \mathrm{T}(\mathrm{u}+\mathrm{v}, \mathrm{u}+\mathrm{v})=\mathcal{Q}_{g_{F}}^{f}(\mathrm{v})$. Thus, $\mathcal{Q}_{g_{F}}^{f}$ is positive semi-definite if and only if $\mathrm{T}(\mathrm{w}, \mathrm{w}) \geq 0$ for any vector $\mathrm{w} \in T M$.

Theorem 3.12. Let $M=I_{f} \times F$ be a standard static space-time with the metric $g=-f^{2} \mathrm{~d} t^{2} \oplus g_{F}$.

(1) If $\mathcal{L}_{g_{F}}^{*} f$ is negative (respectively, positive) semi-definite and $\tau_{g_{F}}$ is nonnegative (respectively, nonpositive), then $\mathrm{T}(\mathrm{w}, \mathrm{w}) \geq 0$ (respectively, $\leq 0)$ for any causal vector $\mathrm{w} \in T M$.

(2) If $\mathcal{L}_{g_{F}}^{*} f \equiv 0$, then $8 \pi \mathrm{T}(\mathrm{w}, \mathrm{w})=-\frac{1}{2} \tau_{g_{F}} g(\mathrm{w}, \mathrm{w})$ for any $\mathrm{w} \in T M$. Thus, $\mathrm{T}(\mathrm{w}, \mathrm{w}) \geq 0$ for any causal vector $\mathrm{w} \in$ TM if and only if $\tau_{g_{F}} \geq 0$.

Remark 3.13. There have been strong and intense studies about the topological significance of the scalar curvature in Riemannian manifolds (see for instance among many others $[20,22,59,60]$ ) since 1965. In particular, Kazdan and Warner classified the compact connected manifolds of dimension $\geq 3$ in three groups [60]:

(1) Those $N$ that admit a metric $h$ with scalar curvature $S_{h} \geq 0$ (non-identically 0 ).

Thus any function is the scalar curvature of some Riemannian metric.

(2) Those $N$ that admit no metric with positive scalar curvature, but do have a metric with $h \equiv 0$.

So a function is the scalar curvature of some Riemannian metric if and only if it is negative somewhere or is identically 0.

(3) The other manifolds, so for any metric $h$, the scalar curvature $S_{h}$ is negative somewhere.

Thus a function is the scalar curvature of some Riemannian metric if and only if it is negative somewhere.

Combining this result with the above Corollaries 3.9 and 3.10, we obtain that the compact manifolds of the third KazdanWarner type cannot be the natural Riemannian part of any standard static space-time verifying the WEC.

Hence, for instance $\left(I \times\left(T^{m} \sharp T^{m}\right),-d t^{2}+h\right)$, where $h$ is a Riemannian metric on the connected sum of two tori $T^{m}$ of dimension $m \geq 3$, never verifies the WEC. Indeed, $T^{m} \sharp T^{m}$ belongs to the third Kazdan-Warner type (see [22]).

We observe that topological obstructions for the problem of prescribed scalar curvature of a Riemannian manifold does not exist for noncompact and connected manifolds, but the situation changes if the completeness of the Riemannian manifold is required (see [22]). Hence it would be interesting to combine these results with the WEC.

Remark 3.14. Since the DEC implies the WEC, it is clear by Corollary 3.9 that $\tau_{g_{F}} \geq 0$ is necessary for the DEC.

Furthermore, recalling that two causal tangent vectors $\mathrm{w}_{1}, \mathrm{w}_{2}$ belong to the same time-cone iff $g\left(\mathrm{w}_{1}, \mathrm{w}_{2}\right) \leq 0$ (see [2, p. 143]) and applying (3.8) it is easy to prove the following theorem.

Theorem 3.15. Let $M=I_{f} \times F$ be a standard static space-time with the metric $g=-f^{2} \mathrm{~d} t^{2} \oplus g_{F}$.

(1) If $\mathscr{L}_{g_{F}}^{*} f \equiv 0$, then $T=-\frac{1}{2} \tau_{g_{F}} g$. Thus $\mathrm{T}\left(\mathrm{w}_{1}, \mathrm{w}_{2}\right) \geq 0$ for all causal vectors $\mathrm{w}_{1}, \mathrm{w}_{2} \in T\left(I_{f} \times F\right)$ in the same time-cone if and only if $\tau_{g_{F}} \geq 0$.

(2) If $\left(F, g_{F}\right)$ is Ricci flat, then for any $\mathrm{u}_{1}, \mathrm{u}_{2} \in T I$ and $\mathrm{v}_{1}, \mathrm{v}_{2} \in T F$, we have $8 \pi \mathrm{T}\left(\mathrm{u}_{1}+\mathrm{v}_{1}, \mathrm{u}_{2}+\mathrm{v}_{2}\right)=\frac{1}{f} Q_{g_{F}}^{f}\left(\mathrm{v}_{1}, \mathrm{v}_{2}\right)$. Thus, $\mathrm{T}\left(\mathrm{w}_{1}, \mathrm{w}_{2}\right) \geq 0$ for any causal vectors $\mathrm{w}_{1}, \mathrm{w}_{2} \in T M$ in the same time-cone iff $\mathrm{Q}_{\mathrm{g}_{F}}^{f} \equiv 0$ iff $\mathscr{L}_{\mathrm{g}_{F}}^{*} f \equiv 0$. 
Remark 3.16. To prove (2) in Theorem 3.15, it is useful to note that for any $\mathrm{v}_{1} \in T F$, the vector given by $\mathrm{w}_{1}:=r_{1} f^{-1}$ $\sqrt{g_{F}\left(\mathrm{v}_{1}, \mathrm{v}_{1}\right)} \partial_{t}+\mathrm{v}_{1} \in T M$ is causal iff $|r| \geq 1$. Moreover, for any $\mathrm{v}_{2} \in T F$, there exists $r_{2} \in \mathbb{R}$ such that $\mathrm{w}_{2}:=r_{2} f^{-1}$ $\sqrt{g_{F}\left(v_{1}, v_{1}\right)} \partial_{t}+v_{2} \in T M$ is causal (as for $v_{1}$ it is sufficient to take $\left.\left|r_{2}\right| \geq 1\right)$ and $g\left(w_{1}, w_{2}\right)=-r_{1} r_{2} \sqrt{g_{F}\left(v_{1}, v_{1}\right)} \sqrt{g_{F}\left(v_{2}, v_{2}\right)}+$ $g_{F}\left(v_{1}, v_{2}\right) \leq 0$, i.e., $w_{2}$ belongs to the same time-cone as $\mathrm{w}_{1}$ does. Indeed, it is sufficient to take $r_{2}$ such that $r_{1} r_{2} \geq \cos _{g_{F}} \widehat{v_{1} v_{2}}$.

Corollary 3.17. Let $M=I_{f} \times F$ be a standard static space-time with the metric $g=-f^{2} \mathrm{~d} t^{2} \oplus g_{F}$. If $\mathscr{L}_{g_{F}}^{*} f \equiv 0$ and $\tau_{g_{F}} \geq 0$, then the DEC is satisfied.

Remark 3.18. Notice that by the polarization formula for a bilinear form,

$$
\mathscr{L}_{g_{F}}^{*} f \equiv 0
$$

is equivalent to

$$
\mathcal{L}_{g_{F}}^{*} f \equiv 0 .
$$

So the hypotheses in Theorems 3.12 and 3.15 and Corollary 3.17 are closely connected to the study of the kernel of the operator $\mathscr{L}_{g_{F}}^{*}$ on suitable Banach spaces. The latter question was studied by several authors; see the references in Remark 2.8 .

In particular, if $f$ is a solution of (3.10), then

$$
\operatorname{tr}_{g_{F}} \mathscr{L}_{g_{F}}^{*} f:=-(s-1) \Delta_{g_{F}} f-\tau_{g_{F}} f=0 .
$$

Thus (3.10) takes the form

$$
\mathscr{L}_{g_{F}}^{*} f=-f \operatorname{Ric}_{g_{F}}+\frac{\tau_{g_{F}}}{s-1} f g_{F}+H_{g_{F}}^{f}=0 .
$$

In $[15$, p. $228-230]$ and $[16$, p. $38-39]$ the authors proved that if $\left(F, g_{F}\right)$ is compact and $f \not \equiv 0$ is a solution of $(3.10)$, then the scalar curvature $\tau_{g_{F}}$ is constant (see [32] also). So, by (3.12) and the well known results about the spectrum of the Laplace-Beltrami operator on a compact Riemannian manifold without boundary (see [21]), there results:

if $\left(F, g_{F}\right)$ is compact and $f \in C_{>0}^{\infty}(F)$, then $f$ verifies (3.10) if and only if $f$ is constant and $\left(F, g_{F}\right)$ is Ricci flat.

On the other hand, in [32, Proposition 2.7] the author proved that (see also [18]):

$f \not \equiv 0$ satisfies (3.10) if and only if the warped product metric $-f^{2} d t^{2}+g_{F}$ is Einstein,

which is also easy to conclude from (2.1).

We remark also the importance of Eq. (3.10) in the study of static Killing Initial Data (static KIDs for short) in the recent articles of R. Beig, P.T. Chruściel, R. Schoen, D. Pollack and F. Pacard [61-63].

Now, under additional hypotheses about the sign of the Ricci curvature, we give a "partial" extension of the Bourguignon/Fischer-Marsden result mentioned above ([15, p. 228-230] and [16]) to the case where the involved manifold is complete but noncompact. Notice that the following three statements are supported by the Liouville type results of P. Li and S.-T. Yau on complete but noncompact Riemannian manifolds.

Theorem 3.19. Let $\left(F, g_{F}\right)$ be a complete Riemannian manifold without boundary where $\operatorname{dim} F \geq 2$. Suppose that the Ricci curvature of $\left(F, g_{F}\right)$ is nonnegative. Given $f \in C_{>0}^{\infty}(F), f$ is a solution of (3.10) if and only if $f$ is constant and $\left(F\right.$, $\left.g_{F}\right)$ is Ricci flat.

Proof. This is a consequence of (3.14) and [18, Corollary 4.9].

Corollary 3.20. Let $\left(F, g_{F}\right)$ be a complete Riemannian manifold without boundary where $\operatorname{dim} F \geq 2$. Suppose that $\left(F, g_{F}\right)$ is not Ricci flat. Then (3.10) admits a nonconstant strictly positive solution only if the Ricci curvature of $\left(F, g_{F}\right)$ is negative somewhere.

Corollary 3.21. Let $\left(F, g_{F}\right)$ be a complete Riemannian manifold without boundary of $\operatorname{dim} F \geq 2$ which is "either compact or complete with nonnegative Ricci curvature" and also let $M=I_{f} \times F$ be a standard static space-time with the metric $g=-f^{2} \mathrm{~d} t^{2} \oplus g_{F}$, where $f \in C_{>0}^{\infty}(F)$. Then, the following properties are equivalent:

(1) the energy-momentum tensor of $M$ and $\tau_{g_{F}}$ are identically zero,

(2) $f$ is a solution of (3.10),

(3) $f$ is a constant and $M$ is Ricci flat,

(4) Ric $_{g_{F}} \equiv 0$ and $Q_{g_{F}}^{f} \equiv 0$,

(5) $f$ is a constant and Ric $_{g_{F}} \equiv 0$,

where $\mathrm{Ric}_{g_{F}}, Q_{g_{F}}^{f}$ and $\tau_{g_{F}}$ are as above. In such cases, DEC (and consequently WEC) is trivially verified; indeed the energymomentum tensor of $M$ is identically zero.

Proof. This is a consequence of (3.13), Theorem 3.19, (2.1) and (3.8). 


\section{Consequences and examples}

In this section, unless otherwise stated, we will assume $\operatorname{dim} F=s \geq 2$.

\subsection{Conformal hyperbolicity}

Before we state our main results, we briefly recall the definition of the Lorentzian pseudo-distance on a Lorentzian manifold $\left(M, g_{M}\right)$ due to Markowitz and then recall some of its elementary properties (see [17] for further details).

Consider the open interval $(-1,1)$ furnished with the Poincaré metric

$$
\mathrm{d} r_{(-1,1)}^{2}=\frac{\mathrm{d} u^{2}}{\left(1-u^{2}\right)^{2}}
$$

So the Poincaré distance between two points $u_{0}, u_{1} \in(-1,1)$ can be expressed as

$$
\rho\left(u_{0}, u_{1}\right)=\frac{1}{2}\left|\log \left(\frac{1+u_{1}}{1-u_{1}} \frac{1-u_{0}}{1+u_{0}}\right)\right| .
$$

Suppose $\gamma$ is a null pre-geodesic in $\left(M, g_{M}\right)$, i.e., $g\left(\gamma^{\prime}, \gamma^{\prime}\right)=0$ and $\gamma^{\prime \prime}=\varphi \gamma^{\prime}$, for some function $\varphi$. Then there is a parameter, called the affine parameter, $r$ for which the null pre-geodesic becomes a null geodesic. Indeed, $r$ is the solution of the following ordinary differential equation $\varphi=r^{\prime \prime} / r$. In this case, a projective parameter $p$ is defined to be a solution of

$$
\{p ; r\}:=\frac{1}{2} \frac{p^{\prime \prime \prime}}{p^{\prime}}-\frac{3}{4}\left(\frac{p^{\prime \prime}}{p^{\prime}}\right)^{2}=-\frac{1}{n-2} \operatorname{Ric}_{M}\left(\gamma^{\prime}(r), \gamma^{\prime}(r)\right),
$$

where $r$ is an affine parameter for a null pre-geodesic of the form given above, $\{p ; r\}$ is the so-called Schwarzian derivative of $p$ respect to $r$ (see [64]) and $\mathrm{Ric}_{M}$ is the Ricci tensor of the Lorentzian manifold $\left(M, g_{M}\right)$. The parameter $r$ is independent of the affine parameter along $\gamma$.

A chain of null geodesic segments joining $p$ to $q$ is:

- a sequence of points $p=p_{0}, p_{1}, \ldots, p_{k}=q$ in $M$,

- pairs of points $\left(a_{1}, b_{1}\right), \ldots,\left(a_{k}, b_{k}\right)$ in $(-1,1)$ and

- projective maps (i.e., a projective map is simply a null geodesic with the projective parameter as the natural parameter) $f_{1}, \ldots, f_{k}$ from $(-1,1)$ into $M$ such that $f_{i}\left(a_{i}\right)=p_{i-1}$ and $f_{i}\left(b_{i}\right)=p_{i}$ for $i=1, \ldots, k$.

The length of such a chain is defined as $L(\alpha)=\sum_{i=1}^{k} \rho\left(a_{i}, b_{i}\right)$.

By combining all the ingredients defined above, Markowitz defines his version of intrinsic Lorentzian pseudo-distance $d_{M}: M \times M \rightarrow[0, \infty)$ as

$$
d_{M}(p, q):=\inf _{\alpha} L(\alpha),
$$

where the infimum is taken over all the chains of null geodesic segments $\alpha$ joining $p$ to $q$. Note that $d_{M}$ is indeed a pseudodistance. The Lorentzian manifold $\left(M, g_{M}\right)$ is said to be conformally hyperbolic when the Lorentzian pseudo-distance $d_{M}$ is a true distance.

We now reproduce the statements of three theorems from [17] which will be useful later.

[17, Theorem 5.1] Let $(M, g)$ be a null geodesically complete Lorentzian manifold. If $(M, g)$ satisfies the curvature condition $\operatorname{Ric}_{M}(\mathrm{x}, \mathrm{x}) \leq 0$ for all null vectors $\mathrm{x}$, then it has a trivial Lorentzian pseudo-distance, i.e., $d_{M} \equiv 0$.

[17, Theorem 5.8] Let $(M, g)$ be an $n(\geq 3)$-dimensional Lorentzian manifold. If $(M, g)$ satisfies the NCC and the null generic condition, NGC for short, (i.e., $\operatorname{Ric}\left(\gamma^{\prime}, \gamma^{\prime}\right) \neq 0$, for at least one point of each inextendible null geodesic $\gamma$ ) then, it is conformally hyperbolic.

[17, Theorem 7.1] The group of conformal automorphisms of a conformally hyperbolic Lorentzian manifold $(M, g)$ has a compact isotropy group at each point $p$.

We will recall some examples in [17].

- Complete Einstein space-times (in particular, Minkowski, de Sitter and the anti-de Sitter space-times) have trivial Lorentzian pseudo-distances because of Theorem 5.1 of [17].

- The Einstein static universe has also trivial Lorentzian pseudo-distance since the space-times in the previous item can be conformally imbedded in the Einstein static universe.

- A Robertson-Walker space-time (i.e., an isotropic homogeneous space-time) is conformally hyperbolic due to [17, Theorem 5.9]. 
- The Einstein-de Sitter space $M$ is conformally hyperbolic and for the null separated points $p$ and $q$, the Lorentzian pseudodistance is given by

$$
d_{M}(p, q)=\frac{5}{4} \log r(p, q)
$$

where $r(p, q)$ denotes the cosmological frequency ratio (see [65] for the explicit computation). The conformal distance between two causally related events in the Einstein-de Sitter space is given as

$$
d_{M}(x, y)=\frac{1}{2}\left|\log \frac{\tau(x)}{\tau(y)}\right|,
$$

where $M=(0, \infty) \times t^{2 / 3} \mathbb{R}^{3}$ with $g=-\mathrm{d} t^{2} \oplus t^{4 / 3} \mathrm{~d} \sigma^{2}$ and $\tau=3 t^{1 / 3}$ (see [65, Theorem 5]).

By applying Theorem 3.7 and the previously restated results of Markowitz, we obtain the theorems that follow.

Theorem 4.1. Let $M=\mathbb{R}_{f} \times F$ be a standard static space-time with the metric $g=-f^{2} \mathrm{~d} t^{2} \oplus g_{F}$. Suppose that $\mathcal{L}_{g_{F}}^{*} f$ is positive semi-definite (this condition is satisfied for example, if $\mathcal{R i c}_{g_{F}}$ and $\mathcal{Q}_{g_{F}}^{f}$ are negative semi-definite). If at least one of the following conditions is verified:

(1) $\left(F, g_{F}\right)$ is compact,

(2) $\left(F, g_{F}\right)$ is complete and $0<\inf f$,

then the Lorentzian pseudo-distance $d_{M}$ on the standard static space-time $(M, g)$ is trivial, i.e., $d_{M} \equiv 0$.

Proof. By Theorem 3.7 and (2.1), the hypothesis for $\mathcal{L}_{g_{F}}^{*} f$ being positive semi-definite implies the RNCC. In both cases the null geodesic completeness of the underlying standard static space-times is a consequence of [66, Theorem 3.12]. So, by $\left[17\right.$, Theorem 5.1], $\mathbb{R}_{f} \times F$ has a trivial Lorentzian pseudo-distance, i.e., $d_{M} \equiv 0$.

Theorem 4.2. Let $M=I_{f} \times F$ be a standard static space-time with the metric $g=-f^{2} \mathrm{~d} t^{2} \oplus g_{F}$. Then, $(M, g)$ is conformally hyperbolic if at least one of the following properties is satisfied:

(1) $\mathcal{L}_{g_{F}}^{*} f$ is negative semi-definite and the NGC is verified,

(2) $\mathcal{R i c}_{g_{F}}$ is positive semi-definite and $\mathcal{Q}_{g_{F}}^{f}$ is positive definite.

Proof. As above, Theorem 3.7 and (2.1), together with the negative semi-definiteness of $\mathcal{L}_{g_{F}}^{*} f$ or positive semi-definiteness of " $\mathcal{R} i c_{g_{F}}$ and $\mathcal{Q}_{g_{F}}^{f}$ " imply the NCC. In (2), the positive definiteness of $\mathcal{Q}_{g_{F}}^{f}$ infers the NGC. Hence, in both cases all the assumptions of [17, Theorem 5.8] are verified and as consequence $\mathbb{R}_{f} \times F$ is conformally hyperbolic.

\subsection{Conjugate points}

We now state some results relating the conformal hyperbolicity and causal conjugate points of a standard static space-time by using [67-70] and also [1]. In [70, Theorem 2.3], it was shown that if the line integral of the Ricci tensor along a complete causal geodesic in a Lorentzian manifold is positive, then the complete causal geodesic contains a pair of conjugate points.

Assume that $\gamma=(\alpha, \beta)$ is a complete causal geodesic in a standard static space-time of the form $M=I_{f} \times F$ with the metric $g=-f^{2} \mathrm{~d} t^{2} \oplus g_{F}$. Then by using $g\left(\gamma^{\prime}, \gamma^{\prime}\right) \leq 0$ and (2.1) we have,

$$
\operatorname{Ric}\left(\gamma^{\prime}, \gamma^{\prime}\right)=-\frac{1}{f} \mathscr{L}_{g_{F}}^{*} f\left(\beta^{\prime}, \beta^{\prime}\right)-\underbrace{g\left(\gamma^{\prime}, \gamma^{\prime}\right)}_{\leq 0} \frac{1}{f} \Delta_{g_{F}} f .
$$

We can easily state the following existence result for conjugate points of complete causal geodesics in a conformally hyperbolic standard static space-time by Theorem 4.2 and [70, Theorem 2.3].

Corollary 4.3. Let $M=I_{f} \times F$ be a standard static space-time with the metric $g=-f^{2} \mathrm{~d} t^{2} \oplus g_{F}$. Then, $(M, g)$ is conformally hyperbolic and any complete causal geodesic in $(M, g)$ has a pair of conjugate points, if at least one of the following properties is satisfied:

(1) $\mathcal{L}_{g_{F}}^{*} f$ is negative definite, $f$ is sub-harmonic and the NGC is verified,

(2) $\mathcal{R i c}_{g_{F}}$ is positive semi-definite and $\mathcal{Q}_{g_{F}}^{f}$ is positive definite.

We will give an existence result for conjugate points of time-like geodesics in a standard static space-time, which will also be conformally hyperbolic because of Theorem 4.2 if one replaces the positive semi-definiteness of $Q_{g_{F}}^{f}$ by the positive definiteness.

In the next corollary (announced in [14]), $\mathbf{L}$ denotes the usual time-like Lorentzian length and diam $\mathbf{L}_{\mathbf{L}}$ denotes the corresponding time-like diameter (see [1, Chapters 4 and 11]). 
Corollary 4.4. Let $M=I_{f} \times F$ be a standard static space-time with the metric $g=-f^{2} \mathrm{~d} t^{2} \oplus g_{F}$. Suppose that $\mathcal{R i c}_{g_{F}}$ and $\mathcal{Q}_{g_{F}}^{f}$ are positive semi-definite. If there exists a constant c such that $\frac{1}{f} \Delta_{g_{F}} f \geq c>0$, then:

(1) any time-like geodesic $\gamma:\left[r_{1}, r_{2}\right] \rightarrow M$ in $(M, g)$ with $\mathbf{L}(\gamma) \geq \pi \sqrt{\frac{n-1}{c}}$ has a pair of conjugate points,

(2) for any time-like geodesic $\gamma:\left[r_{1}, r_{2}\right] \rightarrow M$ in $(M, g)$ with $\mathbf{L}(\gamma)>\pi \sqrt{\frac{n-1}{c}}, r=r_{1}$ is conjugate along $\gamma$ to some $r_{0} \in\left(r_{1}, r_{2}\right)$, and consequently $\gamma$ is not maximal,

(3) if $I=\mathbb{R},\left(F, g_{F}\right)$ is complete and $\sup f<\infty$, then

$$
\operatorname{diam}_{\mathbf{L}}(M, g) \leq \pi \sqrt{\frac{n-1}{c}} .
$$

Proof. First of all we observe that (2.1) implies

$$
\operatorname{Ric}(\mathrm{u}+\mathrm{v}, \mathrm{u}+\mathrm{v}) \geq \frac{\Delta_{g_{F}} f}{f} \geq c>0,
$$

for any unit time-like tangent vector $\mathrm{u}+\mathrm{v}$ on $M$, where $\mathrm{u} \in T I$ and $\mathrm{v} \in T F$.

(1) It is an immediate consequence of (4.1) and [1, Proposition 11.7].

(2) It is an immediate consequence of (4.1) and [1, Proposition 11.8].

(3) In [12, Corollary 3.17] the author proves "if $\left(F, g_{F}\right)$ is a complete Riemannian manifold and $\sup f<\infty$, then $\mathbb{R}_{f} \times F$ is globally hyperbolic". So by applying (4.1) and [1, Theorem 11.9], (3) is obtained.

Remark 4.5. (i) Notice that Corollary 4.4 holds if the positive semi-definiteness of $\mathcal{R} i c_{g_{F}}$ and $\mathcal{Q}_{g_{F}}^{f}$ is replaced by the negative semi-definiteness of $\mathscr{L}_{\mathrm{g}_{\mathrm{f}}}^{*} f$.

(ii) On the other hand, by using [17, Theorem 7.1], one can also deduce that the group of conformal automorphisms of the underlying standard static space-time has a compact isotropy group at each point $p$ when the hypothesis in Theorem 4.2 or Corollary 4.3 is verified.

Remark 4.6. We observe that in Theorems 3.7 and 4.2 and Corollaries 4.3 and 4.4, when the semi-definite positiveness of $Q_{g_{F}}^{f}$ is considered, necessarily $\Delta_{g_{F}} f \geq 0$ (i.e., the function $f$ is sub-harmonic) if $\operatorname{dim} F \geq 2$ (see Remark 2.7). Consequently, in all these statements, if the Riemannian manifold $\left(F, g_{F}\right)$ is compact of dimension at least 2 , then $f$ turns out to be a positive constant (see Remark 2.7).

Thus, the case of a noncompact Riemannian manifold $\left(F, g_{F}\right)$ is particularly relevant. In order to show that the considered assumptions are nonempty (see the discussion about the existence of sub-harmonic functions on complete Riemannian manifolds in Subsection 3.2), we will provide the following simple example:

$$
\begin{aligned}
& \mathbb{R}^{s} \text { with the usual Euclidean metric } g_{0} \\
& M=I \times \Omega, \Omega \subseteq \mathbb{R}^{s} \text { open in } \mathbb{R}^{s} \text { with the induced metric } g_{0} \\
& f: \Omega \rightarrow \mathbb{R} ; \quad f(x)=\frac{1}{2}|x|_{g_{0}}^{2}+\epsilon>0, \quad \epsilon>0 \\
& g=-f^{2} \mathrm{~d} t^{2}+g_{0} \\
& \mathrm{H}_{\Omega}^{f}=g_{0} \\
& \Delta_{\Omega} f \equiv s>0 \\
& \operatorname{Ric}_{\Omega} \equiv 0 .
\end{aligned}
$$

Thus, the assumptions of Theorems 3.7 and 4.2 and Corollary 4.3 are verified. Indeed,

(i) For any $x \in \Omega$, the quadratic form $-\mathscr{L}_{g_{0}}^{*} f=Q_{\Omega}^{f}=(s-1)|\cdot|_{g_{0}}^{2}$ is positive definite on $T_{x} \Omega=\mathbb{R}^{s}$.

(ii) $\operatorname{Ric}_{\Omega} \equiv 0$.

If we suppose that $\Omega$ is bounded, then the assumptions of Corollary 4.4 are verified with the exception of item 3 (i.e., the completeness of $\left.\left(F, g_{F}\right)\right)$. In this case, there exists a constant $c>0$ such that

$$
\frac{1}{f} \Delta_{\Omega} f=\frac{s}{\frac{1}{2}|x|^{2}+\epsilon} \geq c>0 .
$$




\subsection{Some special examples}

Now, we will concentrate our attention on some special families of metrics that allow an easier control of the sign of $\mathcal{Q}_{g_{F}}^{f}$. For these metrics, there is a strong relation between the Riemannian metric $g_{F}$ and the function $f \in C_{>0}^{\infty}(F)$.

"Concircular scalar fields". We follow the terminology used in [71], i.e., we will call a nonconstant scalar field $u$ on $F$ a concircular scalar field if it satisfies the equation

$$
\mathrm{H}_{g_{F}}^{u}=\phi g_{F}
$$

where $\phi$ is a scalar field called the characteristic function of $u$.

Notice that in this situation, taking the $g_{F}$-trace, we obtain

$$
\Delta_{g_{F}} u=\phi s
$$

and as a consequence,

$$
Q_{g_{F}}^{u}=(s-1) \phi g_{F} .
$$

Thus, being $\operatorname{dim} F \geq 2$, the $\operatorname{sign}$ of $Q_{g_{F}}^{u}$ is determined by $\operatorname{sign} \phi$.

We observe that Eq. (4.3) admits a nonconstant solution with a smooth function $\phi$ of constant sign only if the Riemannian manifold $\left(F, g_{F}\right)$ is noncompact. Thus, there exists no concircular scalar field with a characteristic function of constant sign on a compact Riemannian manifold.

Thus, applying Theorems 4.1 and 4.2, respectively, we state the results that follow.

Corollary 4.7. Let $\left(F, g_{F}\right)$ be such that $\mathcal{R i c}_{g_{F}}$ is negative semi-definite. Suppose that $\left(F, g_{F}\right)$ admits a positive concircular scalar field $u$ with a nonpositive characteristic function $\phi$. If $\left(F, g_{F}\right)$ is complete and $0<\inf u$, then the Lorentzian pseudo-distance $d_{M}$ on the standard static space-time $M=I_{u} \times F$ with the metric $g=-u^{2} \mathrm{~d} t^{2} \oplus g_{F}$ is trivial, i.e., $d_{M} \equiv 0$.

Corollary 4.8. Let $\left(F, g_{F}\right)$ be such that $\mathcal{R i c}_{g_{F}}$ is negative semi-definite. If $\left(F, g_{F}\right)$ admits a positive concircular scalar field $u$ with a positive characteristic function $\phi$, then the standard static space-time $M=I_{u} \times F$ with the metric $g=-u^{2} \mathrm{~d} t^{2} \oplus g_{F}$ is conformally hyperbolic.

"Hessian manifolds". This type of manifold is closely related to the previously defined concircular scalar fields. We begin by recalling the definition of Hessian manifolds (see for instance the recent articles [72-76], and in particular, the recent book by H. Shima [77]).

First of all, we remark a notational matter: we say that a connection $D$ on a manifold $N$ is $S$-flat if its torsion and curvature tensor vanish identically. We also say that a manifold $N$ endowed with an $S$-flat connection $D$ is an $S$-flat manifold and we denote it by $(N, D)$. Notice that in [77], S-flat manifolds are called flat. On the other hand, according to our convention, the concept of flatness only means that the curvature tensor vanishes identically.

A Riemannian metric $h$ on an $S$-flat manifold $(N, D)$ is said to be a locally (respectively, globally) Hessian metric if $h$ is locally (respectively, globally) expressed by the Hessian, i.e., $h=D d u$, where $u$ is a local (respectively, global) smooth function on $N$. Such a pair $(D, h)$ is called a locally (respectively, globally) Hessian structure on $N$, and $u$ is said to be a local (respectively, global) potential of $(D, h)$. A manifold $N$ provided with a locally (respectively, globally) Hessian structure is called a locally (respectively, globally) Hessian manifold and is denoted by $(N, D, h)$.

If it is necessary, we will say that $N$ is $u$-globally Hessian to indicate the specific function $u \in C^{\infty}(N)$ such that $h=D d u$ on the entire manifold $N$.

It is clear that a globally Hessian manifold is also locally Hessian.

In [77], it is proved that if a Riemannian manifold $(N, h)$ is flat with respect to the Levi-Civita connection $\nabla$, then it is also a locally Hessian manifold with the Hessian structure $(\nabla, h)$. Notice that for the Levi-Civita connection both concepts, i.e., flatness and S-flatness coincide.

Moreover, in [77] several important examples of locally and globally Hessian structures are provided. Here, we mention a more classical and well known one, namely $\mathbb{R}^{s}$ furnished with the usual canonical flat connection. More explicitly, $\mathbb{R}^{s}$ is a flat $u$-globally Hessian manifold where $u(x)=\frac{1}{2}|x|_{g_{0}}^{2}$, where $g_{0}$ is the canonical Euclidean metric on $\mathbb{R}^{s}$.

Let $(N, h)$ be a Riemannian manifold with the Levi-Civita connection $\nabla$. Then, $(\nabla, h)$ is a globally Hessian structure on $N$ with positive global potential if and only if $(N, h)$ is flat and admits a positive concircular scalar field with characteristic function $\phi \equiv 1$. In this case, the positive concircular scalar field can be taken as the positive global potential, and vice versa. Notice that when we say $(\nabla, h)$ is a globally Hessian structure on $N$ with positive global potential, this particularly implies that the global potential is nonconstant (since $h$ is a metric). Thus, applying Corollary 4.8 results:

Corollary 4.9. Let $\left(F, g_{F}\right)$ be a $u$-globally Hessian manifold with the Hessian structure $\left(\nabla^{g_{F}}\right.$, $\left.g_{F}\right)$, where the global potential $u \in C_{>0}^{\infty}(F)$ and $\nabla^{g_{F}}$ denotes the Levi-Civita connection of $g_{F}$. Then, the standard static space-time of the form $M=I_{u} \times F$ with the metric $g=-u^{2} \mathrm{~d} t^{2} \oplus g_{F}$ is conformally hyperbolic.

Remark 4.10. We will note some facts related to the previous statements and examples of this section. 
(i) Now let $(D, h)$ be a locally Hessian structure on a manifold $N$ of dimension $s$ and $\nabla$ be the Levi-Civita connection associated to $h$. In [75], necessary and sufficient conditions are given to have $\nabla=D$, when $N$ is a compact manifold without boundary.

Note that in this case, i.e., $h=D \mathrm{~d} u=\nabla \mathrm{d} u$, we necessarily have $\Delta u=s$. Indeed, it is sufficient to take the $h$-trace of both sides.

Furthermore, if the Hessian structure is $u$-global, then $u$ is a function with a Laplacian of constant sign on a compact Riemannian manifold. Hence, $u$ is a constant (see Remark 2.7).

In particular, this shows that the relevant cases for Corollary 4.9 are those in which $\left(F, g_{F}\right)$ is assumed to be noncompact. Indeed, if it is compact, then the assumptions of the last corollary are not verified.

One simple example of this situation is the Riemannian manifold (i.e., globally Hessian manifold $)\left(F, g_{F}\right)=\left(\Omega, g_{0}\right)$ where $\Omega$ is an open subset of $\mathbb{R}^{s}$ considered in Remark 4.6.

If we further add the completeness assumption for $\left(F, g_{F}\right)$ to the hypothesis of Corollary 4.9 , then $\left(F, g_{F}\right)$ becomes a Euclidean space. Indeed, this is a consequence (for instance) of [71, Theorem $2(I, B)]$.

(ii) If $\left(F, g_{F}\right)$ is a complete Riemannian manifold of dimension 1 and $u \in C_{>0}^{\infty}(F)$ verifying $0<$ inf $u$, then the Lorentzian pseudo-distance $d_{M}$ on a standard static space-time of the form $M=I_{u} \times F$ with metric $g=-u^{2} \mathrm{~d} t^{2} \oplus g_{F}$ is trivial i.e., $d_{M} \equiv 0$. Indeed, $\operatorname{dim} F=s=1$ implies that $Q_{g_{F}}^{u} \equiv 0$ (by the definition of $Q_{g_{F}}^{u}$ ); so we can directly apply Theorem 4.1.

By means of analogous arguments, the hypothesis stating that " $u$ is a concircular scalar field" in Corollary 4.7 turns out to be irrelevant when the dimension of the fiber is 1 .

(iii) By considering the relation (4.4), we can notice that relevant examples for Corollaries 4.7 and 4.8 correspond to the case of having a Riemannian part of dimension at least 2.

For instance, in $\mathbb{R}^{n}$ with the usual metric $g_{0}=\mathrm{Id}_{\mathbb{R}^{n}}$, the existence of a concircular scalar field, i.e., $\mathrm{H}_{\mathbb{R}^{n}}^{u}=\phi g_{0}$, implies that $u(x)=\sum_{i=1}^{n} \xi_{i}\left(x_{i}\right)$ and hence, $\partial_{i i} \xi_{i}\left(x_{i}\right)=\partial_{j j} \xi_{j}\left(x_{j}\right)=\phi(x)$ for any $i \in\{1, \ldots, n\}-\{j\}$. Thus, $\phi=\phi_{0}$ is constant and $\xi_{i}\left(x_{i}\right)=a_{i} x_{i}^{2}+b_{i} x_{i}+c_{i}$, where $\phi_{0}=2 a_{i}$ for any $i$ with the coefficients $a_{i}, b_{i}, c_{i} \in \mathbb{R}$.

Finally, we will mention some simple examples where the suppositions of Corollaries 4.7 and 4.8 are not satisfied. Let $\left(\mathbb{R}^{2}, g_{0}\right)$ be the usual Euclidean plane and $\Omega$ be an open subset of $\mathbb{R}^{2}$. Then $\left(\Omega, g_{0}\right)$ is a Ricci-flat Riemannian manifold. Therefore:

(1) If $\Omega=\mathbb{R}^{2}$, then the hypothesis of Corollary 4.7 cannot be verified. Indeed $\left(\mathbb{R}^{2}, g_{0}\right)$ is complete, but does not admit a positive concircular scalar field $u$ with a nonpositive characteristic function $\phi$ such that $0<\inf u$. This follows from (4.3) and either direct calculus or the classical Liouville nonexistence theorems for nonconstant superharmonic functions bounded from below on $\left(\mathbb{R}^{2}, g_{0}\right)$ (see $\left.[78,79]\right)$.

(2) If $\Omega=\left\{x \in \mathbb{R}^{2}: x_{1}>0, x_{2}>0\right\}$, then the hypothesis of Corollary 4.8 is verified. For example, take $u\left(x_{1}, x_{2}\right)=x_{1}^{2}+x_{2}^{2}+x_{1}+x_{2}+1$ as a concircular scalar field with positive characteristic function 2 .

(3) For any $\Omega$, the hypothesis of Corollary 4.8 is satisfied. For instance, take $u\left(x_{1}, x_{2}\right)=x_{1}^{2}+x_{2}^{2}+1$ as a concircular scalar field with positive characteristic function 2 . Notice that this concircular scalar field is different from the one given in (2).

\section{Conclusions}

Our first goal was to obtain a family of necessary and/or sufficient conditions for a set of energy and convergence conditions on standard static space-times. We state a family of results establishing a connection between the strong energy condition and a family of Liouville type results for sub-harmonic functions on the Riemannian part of a standard static space-time too. We also obtain a set of results about the weak and dominant energy conditions. All these results are based on suitable hypotheses for the definiteness of the quadratic forms associated to the Ricci tensor and the tensors $\mathscr{L}_{g_{F}}^{*} f$ and $Q_{g_{F}}^{f}$ defined in Section 2. Similarly to the independent studies of Bourguignon, Fischer-Marsden or Lafontaine, we observe the importance of the kernel of the operator $\mathscr{L}_{g_{F}}^{*}$, not only in the study of standard static space-times, but also in Riemannian geometry, more specifically in the study of critical points of the scalar curvature map in any dimension. Regarding this matter, taking into account the sign of the Ricci curvature and applying Liouville type results, we provide a statement about the existence of positive functions in the kernel of the operator $\mathscr{L}_{g_{F}}^{*}$ where the involved manifold is complete but noncompact.

The second goal was to apply the previous results together with several results of M.J. Markowitz and D.E. Allison to obtain sufficient conditions for studying the conformal hyperbolicity and the existence of conjugate points on causal geodesics on a standard static space-time.

Finally, we showed some examples and results relating the tensor $Q_{F}^{f}$, conformal hyperbolicity, concircular scalar fields and Hessian manifolds.

\section{Acknowledgements}

The authors wish to thank the referee for the useful and constructive suggestions. F.D. thanks The Abdus Salam International Centre of Theoretical Physics for their warm hospitality where part of this work has been done. 


\section{References}

[1] J.K. Beem, P.E. Ehrlich, K.L. Easley, Global Lorentzian Geometry, 2nd ed., Marcel Dekker, New York, 1996.

[2] B. O'Neill, Semi-Riemannian Geometry with Applications to Relativity, Academic Press, New York, 1983.

[3] O. Kobayashi, M. Obata, Conformally-flatness and static space-time, in: Manifolds and Lie Groups, in: Progress in Mathematics, vol. 14, Birkhäuser, 1981, pp. 197-206.

[4] G.S. Hall, Symmetries And Curvature Structure in General Relativity, in: Lecture Notes in Physics, vol. 46, World Scientific, 2004.

[5] L.P. Hughston, K.P. Tod, Introduction to General Relativity, in: LMS Student Texts, vol. 5, CUP, 1990.

[6] R.K. Sachs, H. Wu, General Relativity for Mathematicians, 1977.

[7] B. Carter, Energy dominance and the Hawking-Ellis vacuum conservation theorem, in: G.W. Gibbons, E.P.S. Shellard, S.J. Rankin (Eds.), The Future of Theoretical Physics and Cosmology, Celebrating Stephen Hawking's 60th Birthday, Cambridge Univ. Press, 2003, pp. 177-184. arXiv:gr-qc/0205010v1.

[8] V.P. Frolov, I.D. Novikov, Black Hole Physics - Basic Concepts and New Developments, Springer, 1998.

[9] S.W. Hawking, G.F.R. Ellis, The Large Scale Structure of Space-Time, Cambridge University Press, UK, 1973.

[10] M. Visser, Lorentzian Wormholes-From Einstein to Hawking, Springer-Verlag, New York, 1996

[11] R.W. Wald, General Relativity, The University of Chicago Press, Chicago, London, 1984.

[12] D.E. Allison, Lorentzian warped products and static space-times, Ph.D. Thesis, University of Missouri-Columbia, 1985.

[13] D.E. Allison, Energy conditions in standard static space-times, Gen. Relativity Gravitation 20 (2) (1988) $115-122$.

[14] F. Dobarro, B. Ünal, Hessian tensor and standard static space-times, in: M. Plaue, M. Scherfner (Eds.), Advances in Lorentzian Geometry, Shaker Verlag, Germany, 2008. http://www.shaker.eu/catalogue/Details.asp?ISBN=978-3-8322-7786-4. arXiv:math.DG/0607113v2.

[15] A.E. Fischer, J.E. Marsden, Linearization stability of nonlinear partial differential equations, Proc. Sympos. Pure Math. 27 (1975) $219-263$.

[16] J.-P. Bourguignon, Une stratification de l'espace des structure Riemannienes, Compos. Math. 30 (1)(1975) 1-41.

[17] M.J. Markowitz, An intrinsic conformal Lorentz pseudodistance, Math. Proc. Cambridge Philos. Soc. 89 (2) (1981) $359-371$.

[18] F. Dobarro, B. Ünal, Special standard static spacetimes, Nonlinear Anal. TMA 59 (5) (2004) 759-770.

[19] F. Dobarro, B. Ünal, Curvature of multiply warped products, J. Geom. Phys. 55 (1) (2005) 75-106.

[20] T. Aubin, Some Nonlinear Problems in Riemannian Geometry, in: Springer Monographs in Mathematics, Springer Verlag, Berlin, 1998

[21] P.H. Bérard, Spectral Geometry: Direct and Inverse Problems, in: Lec. Notes in Math., vol. 1207, Springer-Verlag, Heidelberg, 1986.

[22] L. Bérard Bergery, La courbure scalaire de variété Riemanniennes, Seminaire N. Bourbaki 556 (1980) $225-245$.

[23] K. Yano, Integral Formulas in Riemannian Geometry, Marcel Dekker, New York, 1970.

[24] A. Lichnerowicz, Propagateurs et Commutateurs en relativite generale, Publ. Scientifiques, IHES (10) (1961) 293-344.

[25] A.E. Fischer, J.E. Marsden, Manifolds of Riemannian metrics with prescribed scalar curvature, Bull. Amer. Math. Soc. 80 (3) (1974) $479-484$.

[26] J. Marsden, Applications of Global Analysis in Mathematical Physics, Publish or Perish Inc., 1974.

[27] A.E. Fischer, J.E. Marsden, Deformations of the scalar curvature, Duke Math. J. 42 (1975) 519-547.

[28] O. Kobayashi, M. Obata, Certain mathematical problems on static models in general relativity, in: S.S. Chern, W. Wen-tsün (Eds.), in: Proceedings of the 1980 Beijing Symposium on Differential Geometry and Differential Equations, Vol. 3, 1980, pp. 1333-1344.

[29] O. Kobayashi, A differential equation arising from scalar curvature function, J. Math. Soc. Japan 34 (4) (1982) 665-675.

[30] J. Lafontaine, Sur la Géométrie d'une généralization de l'équation différentielle d'Obata, J. Math. Pures Appl. 62 (1983) $63-72$.

[31] R. Mazzeo, D. Pollack, K. Uhlenbeck, Moduli spaces of singular Yamabe metrics, J. Amer. Math. Soc. 9 (2)(1996).

[32] J. Corvino, Scalar curvature deformation and gluing construction for the Einstein constraint equations, Comm. Math. Phys. 214 (2000) 137-189.

[33] S. Hwang, Critical points of the total scalar curvature functional on the space of metrics of constant scalar curvature, Manuscripta Math. 103 (2000) $135-142$.

[34] J. Lafontaine, L. Rozoy, Courbure scalaire et trous noirs, Sémin. Théor. Spectr. Géom. Grenoble 18 (2000) 69-76.

[35] S. Hwang, The critical point equation on a three-dimensional compact manifold, Proc. Amer. Math. Soc. 131 (10) (2003) 3221-3230.

[36] J. Corvino, R.M. Schoen, On the asymptotics for the vacuum Einstein constraint equations, J. Differential Geom. 73 (2006) $185-217$.

[37] J. Lafontaine, A remark about static spacetimes, J. Geom. Phys. 59 (1) (2009) 50-53.

[38] F. Tipler, Energy conditions and spacetime singularities, Phys. Rev. D 17 (1978) 2521-2528.

[39] A. Borde, Geodesic focusing, energy conditions and singularities, Classical Quantum Gravity 4 (1987) $343-356$.

[40] J.-H. Eschenburg, G.J. Galloway, Lines in space-times, Comm. Math. Phys. 148 (1992) 209-216.

[41] M. Cai, G.J. Galloway, On the topology and area of higher dimensional black holes, Classical Quantum Gravity 18 (2001) 2707-2718. arXiv:hepth/0102149v2.

[42] R. Emparan, H.S. Reall, Black holes in higher dimensions. arXiv:0801.3471.

[43] G.J. Galloway, R. Schoen, A generalization of Hawkings black hole topology theorem to higher dimensions, Comm. Math. Phys. 266 (2006) 571-576.

[44] M. Nardmann, Nonexistence of spacelike foliations and the dominant energy condition in Lorentzian geometry. arXiv:math/0702311v1 [math.DG].

[45] L. Andersson, G.J. Galloway, dS/CFT and spacetime topology, Adv. Theor. Math. Phys. 6 (2002) $307-327$.

[46] T. Frankel, Gravitational Curvature, W. H. Freeman, San Francisco, 1979.

[47] K.K. Lee, Another possible abnormality of compact space-time, Canad. Math. Bull. 18 (1975) 695-697

[48] Y.M. Cho, D.H. Park, Closed time-like curves and weak energy condition, Phys. Lett. B 402 (1997) 18-24.

[49] A. Ori, Y. Soen, Causality violation and the weak energy condition, Phys. Rev. D 49 (1994) 3990-3997.

[50] R. Penrose, Techniques of Differential Topology in Relativity, in: Regional Conference Series in Applied Mathematics, SIAM, PA, 1972.

[51] J.K. Beem, P. Ehrlich, Conformal deformations, Ricci curvature and energy conditions on globally hyperbolic spacetimes, Math. Proc. Cambridge Philos. Soc. 84 (1978) 159-175.

[52] G.W. Gibbons, Causality and the Skyrme model. arXiv:hep-th/0302149v1.

[53] P. Li, Curvature and Function Theory on Riemannian Manifolds, in: Surveys in Differential Geometry: Papers dedicated to Atiyah, Bott, Hirzebruch, and Singer, Vol. VII, International Press, 2000, pp. 375-432.

[54] P. Li, Lectures on Harmonic Functions, University of California, Irvine, 2004. http://www.math.uci.edu/ pli/.

[55] S.T. Yau, Some function-theoretic properties of complete Riemannian manifolds and their applications to geometry, Indiana Math. J. 25 (1976) 659-670.

[56] P. Li, R. Schoen, $L^{p}$ and mean value properties of subharmonic functions on Riemannian manifolds, Acta Math. 153 (1984) $279-301$.

[57] P. Li, Uniqueness of L1 solutions for the Laplace equation and the heat equation on Riemannian manifolds, J. Differential Geom. 20 (1984) $447-457$.

[58] P. Ehrlich, M. Sánchez, Some semi-Riemannian volume comparison theorems, Tohôku Math. J. 52 (2000) $285-314$.

[59] J.L. Kazdan, Prescribing the Curvature of a Riemannian Manifold, in: CBMS Regional Conference Series in Mathematics, vol. 57 , AMS, USA, 1985.

[60] J.L. Kazdan, Applications of Partial Differential Equations to Problems in Geometry, 2006, http://www.math.upenn.edu/ kazdan/.

[61] R. Beig, P.T. Chruściel, R. Schoen, KIDs are non-generic, Ann. Henri Poincaré 6 (2005) 155-194.

[62] P.T. Chruściel, D. Pollack, Singular Yamabe metrics and initial data with exactly Kottler-Schwarzschild-de Sitter ends. arXiv:0710.3365v2 [gr-qc].

[63] P.T. Chruściel, F. Pacard, D. Pollack, Singular Yamabe metrics and initial data with exactly Kottler-Schwarzschild-de Sitter ends II. arXiv:0803.1817v1 [gr-qc]

[64] T. Sasaki, Projective differential geometry and linear homogeneous differential equations, Rokko Lect. Math., Kobe University, Japan 5 (1999) 1-115.

[65] M.J. Markowitz, Conformal hyperbolicity of Lorentzian warped products, Gen. Relativity Gravitation 14 (2)(1982) 1095-1105.

[66] D.E. Allison, Geodesic completeness in static spacetimes, Geom. Dedicata 26 (1988) 85-97.

[67] J.K. Beem, Lorentzian geometry in the large, in: Mathematics of gravitation, Part I (Warsaw, 1996), in: Banach Center Publ., Part I, vol. 41, Polish Acad. Sci., Warsaw, 1997, pp. 11-20. 
[68] J.K. Beem, P. Ehrlich, Cut points, conjugate points and Lorentzian comparison theorems, Math. Proc. Cambridge Philos. Soc. 86 (2) (1979) 365-384

[69] J.K. Beem, P. Ehrlich, Singularities, incompleteness and the Lorentzian distance function, Math. Proc. Cambridge Philos. Soc. 85 (1) (1979) $161-178$.

[70] C. Chicone, P. Ehrlich, Line integration of Ricci curvature and conjugate points in Lorentzian and Riemannian manifolds, Manuscripta Math. 31 (1-3) (1980) 297-316.

[71] Y. Tashiro, Complete Riemannian manifolds and some vector fields, Trans. Amer. Math. Soc. 117 (1965) 251-275.

[72] L.A. Caffarelli, J.A. Viaclovsky, On the regularity of solutions to Monge-Ampère equations on Hessian manifolds, Comm. Partial Differential Equations $26(11-12)(2001) 2339-2351$

[73] J.J. Duistermaat, On Hessian Riemannian structures, Asian J. Math. 15 (1) (2001) 79-91.

[74] H. Shima, Hessian manifolds of constant Hessian sectional curvature, J. Math. Soc. Japan 47 (4) (1995) 735-753.

[75] H. Shima, K. Yagi, Geometry of Hessian manifolds, Differential Geom. Appl. 7 (1997) 277-290.

[76] B. Totaro, The curvature of a Hessian metric, Int. J. Math. 15 (4) (2004) 369-391.

[77] H. Shima, The Geometry of Hessian Structures, World Scientific, 2007.

[78] M.H. Protter, H.F. Weinberger, Maximum Principles in Differential Equations, Springer Verlag, New York, 1984.

[79] J. Serrin, H. Zou, Cauchy-Liouville and universal boundedness theorems for quasilinear elliptic equations and inequalities, Acta Math. 189 (2002) 79-142. 